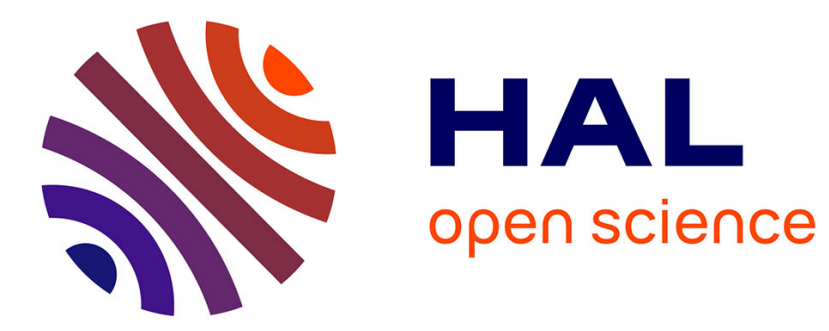

\title{
A qualitative dynamical model for cardiotocography simulation
}

Alfredo Illanes, Michel Haritopoulos, Felipe Robles, Francisco Guerra

\section{To cite this version:}

Alfredo Illanes, Michel Haritopoulos, Felipe Robles, Francisco Guerra. A qualitative dynamical model for cardiotocography simulation. 42nd annual conference of Computing in Cardiology - CinC 2015, Aug 2015, Nice, France. hal-01203112

\section{HAL Id: hal-01203112 \\ https://hal.science/hal-01203112}

Submitted on 22 Sep 2015

HAL is a multi-disciplinary open access archive for the deposit and dissemination of scientific research documents, whether they are published or not. The documents may come from teaching and research institutions in France or abroad, or from public or private research centers.
L'archive ouverte pluridisciplinaire HAL, est destinée au dépôt et à la diffusion de documents scientifiques de niveau recherche, publiés ou non, émanant des établissements d'enseignement et de recherche français ou étrangers, des laboratoires publics ou privés. 


\title{
A qualitative dynamical model for cardiotocography simulation
}

\author{
Alfredo Illanes ${ }^{1}$, Michel Haritopoulos ${ }^{2}$, Felipe Robles ${ }^{1}$, Francisco Guerra ${ }^{3}$ \\ ${ }^{1}$ Facultad de Ciencias de la Ingeniería, Universidad Austral de Chile, Valdivia, Chile \\ ${ }^{2}$ Laboratoire PRISME, Université d'Orléans, Orléans, France \\ ${ }^{3}$ Facultad de Medicina, Universidad Austral de Chile, Valdivia, Chile
}

\begin{abstract}
The purpose of this work is to present a new mathematical model for fetal monitoring simulation. It involves the simultaneous generation of fetal heart rate and maternal uterine contraction signals through a parametrical model. This model allows the generation of the main fetal monitoring dynamics including fetal movements, acceleration and deceleration of the heart rate and the dynamical adjustment of fetal heart rate following an uterine contraction. Simulated tracings were analyzed by specialists and evaluated in terms of signal realism and dynamics. Results show no significant differences between real and computer-generated signals.
\end{abstract}

\section{Introduction}

In current clinical practice the assessment of fetal wellbeing during labor and delivery is commonly performed through a technique for intrapartum fetal surveillance known as cardiotocograph (CTG), which measures continuously the fetal heart rate (FHR) in relation to the maternal uterine contractions (UC) [1]. The CTG gives an indication of the main determinant of the acute fetal condition. Specifically, the CTG is used to assess the adaptation of fetal oxygenation during labor for identifying hypoxic fetuses and preventing metabolic acidemia, which is a serious complication that increases the risk of perinatal mortality and morbidity [1]. However, the interpretation of CTG has been shown to suffer from wide intra- and inter- observer disagreement, leading to a poor interpretation reproducibility, despite the introduction of clinical guidelines for CTG interpretation and evaluation [2,3].

In cardiotocography, as in other areas, simulators and mathematical models can be used to assist clinical staff in the interpretation of the CTG, since they provide a risk-free and controllable environment for training. However, currently available obstetric simulators and training programs, as for example Fetal Sim (http://www.advmedsim.com), iSimulate http://www.isimulate.com/ctgi/, NOEL-
LETM (http://www.gaumard.com), SIMoneTM (http://www.a3bs.com), and SimMom (http: / / www. laerdal.com/la/SimMom\#/), provide limited realism and most of them simulate only static scenarios without the possibility of changing dynamics online during the simulation [4]. This leads to a limited number of scenarios that can be simulated.

In this sense, a dynamical model for fetal monitoring can help to avoid the problem of static scenarios. Several dynamical models has been proposed for UC and FHR signal generation [4-6]. However, most of them do not integrate the dynamical relationships between both signals. Dynamical models relating UC and FHR signals have been proposed mainly in the context of assisting CTG interpretation through system identification approaches $[7,8]$ and for the simulation of specific FHR dynamics [9-11] using complex physiological models.

In this work a new qualitative dynamical model for simulating synthetic cardiotocographic recordings of normal and critical situations in labor and delivery is proposed as a training tool for educational purposes. The model allows simulating the main CTG dynamics including baseline of the FHR, variability, accelerations, decelerations (early, late and variables). The novelty of this simulator lays on a function representing the online fetus welfare status through a simple parametrical model. Moreover, the user can set different simulation parameters, including the possibility of generating an uterine contraction or a fetal movement on-line during the simulation.

Simulated tracings were analyzed by specialists and evaluated in terms of signal realism and dynamics of the normal changes, periodic and episodic changes of the FHR with no significant differences between real and computergenerated CTG tracings.

\section{Materials and methods}

The CTG model simulator involves seven main interconnected blocks, which can be classified in three types (see Figure 1):

1. The user's parameter settings block, called user control 
panel (UCP).

2. Four signal generators blocks, corresponding to a fetal movement (FM) generator, a FHR generator, a UC generator and a deceleration type generator.

3. Two actuators blocks, corresponding to a fetal welfare function generator and a fetal state evaluator. Both blocks allow the control of the FHR signal using a fetal welfare function, which serves for estimating the condition state of the fetus from a well known 5-tier CTG interpretation guideline [12].

The model and the simulator have been implemented using Python 2.7.

\subsection{User control panel (UCP)}

Through this block the user can set the initial conditions of the simulation as the FHR baseline and variance, the simulation time and the average number of contractions and fetal movements in ten minutes. There is also an option for the user to interact online with the model during the simulation by generating a fetal movement or an uterine contraction during the simulation.

Another important parameter to be set by the user is the fetus profile, which corresponds to the degree of complication depending on the degree of asphyxia suffered by the fetus at the beginning of the simulation (as initial condition of the model). This profile can be set in anyone of the four following initial fetus complication states: normal, mild, moderate and severe asphyxia.

\subsection{Fetal movement generator}

This block generates the intensity, duration and onset of a fetal movement (FM). It is based on a set of random variables whose probability distribution function parameters can be set by the user at the beginning of the simulation (number of FM every ten minutes), or during the simulation (FM generated online), or even it can be controlled by other blocks during the simulation (cases when the fetus condition does not allow the fetus to perform a movement).

\subsection{FHR generator}

This block provides a preliminary FHR signal with a specific baseline and variability, but with neither accelerations nor decelerations of the FHR. The acceleration and deceleration dynamics, as well as the online dynamical changes of variability and baseline are controlled mainly by the fetal welfare function and the deceleration type generator, which are presented below.

The preliminary FHR signal is generated by using the dynamical model presented in [5], which allows the generation of RR tachograms through the inverse Fourier transform of a parametrical spectrum model. The spectrum pa- rameters were tuned so as to be adapted to the FHR and their values were inspired from those one can find in the literature $[1,8,9]$.

\subsection{UC Generator}

This block generates the instantaneous UC signal based on the assumption that a maternal uterine contraction has a Gaussian shape. For this purpose the model presented in [4] is used. The signal baseline is generated as the addition of a random variable and a colored low frequency noise. The block is able to generate contractions at random time instants (following a user's input of an average number of contractions every ten minutes) and it can also generate an online contraction triggered by the user during the simulation.

\subsection{Deceleration type generator}

This block generates the type of deceleration according to the standard classification made in CTG guidelines [12]: early, late, variable and no deceleration. Each generated deceleration type is accompanied by a vector of parameters which characterizes the deceleration: onset, depth, duration, delay between contraction and deceleration onsets and the descending and ascending deceleration slopes.

These deceleration parameters depend on the welfare of the fetus (function $w$, cf. Section 2.7), on the last hour's deceleration types (this is denoted in the diagram of the Figure 1 as accumulator) and on the current deceleration type.

\subsection{Fetal state evaluator}

The fetal state evaluator block receives the FHR signal and patterns (variability, baseline, decelerations type) and evaluates and classifies it into the one of the 133 fetal states of the 5-tiers CTG interpretation system presented in [12]. A discretization of these states allows to define one reduced fetal state set, which serves to adjust the parameters of the fetal welfare function presented below.

\subsection{Fetal welfare function generator}

This is the most important part of the model since it allows the dynamical changes in the FHR signal. The main idea behind this block is to generate a function which measures online the condition of the fetus by quantifying its current welfare. This is done through the following first order parametric ODE:

$$
\frac{d w}{d t}=\frac{w_{\infty}(c u)-w}{\tau(c u)}
$$




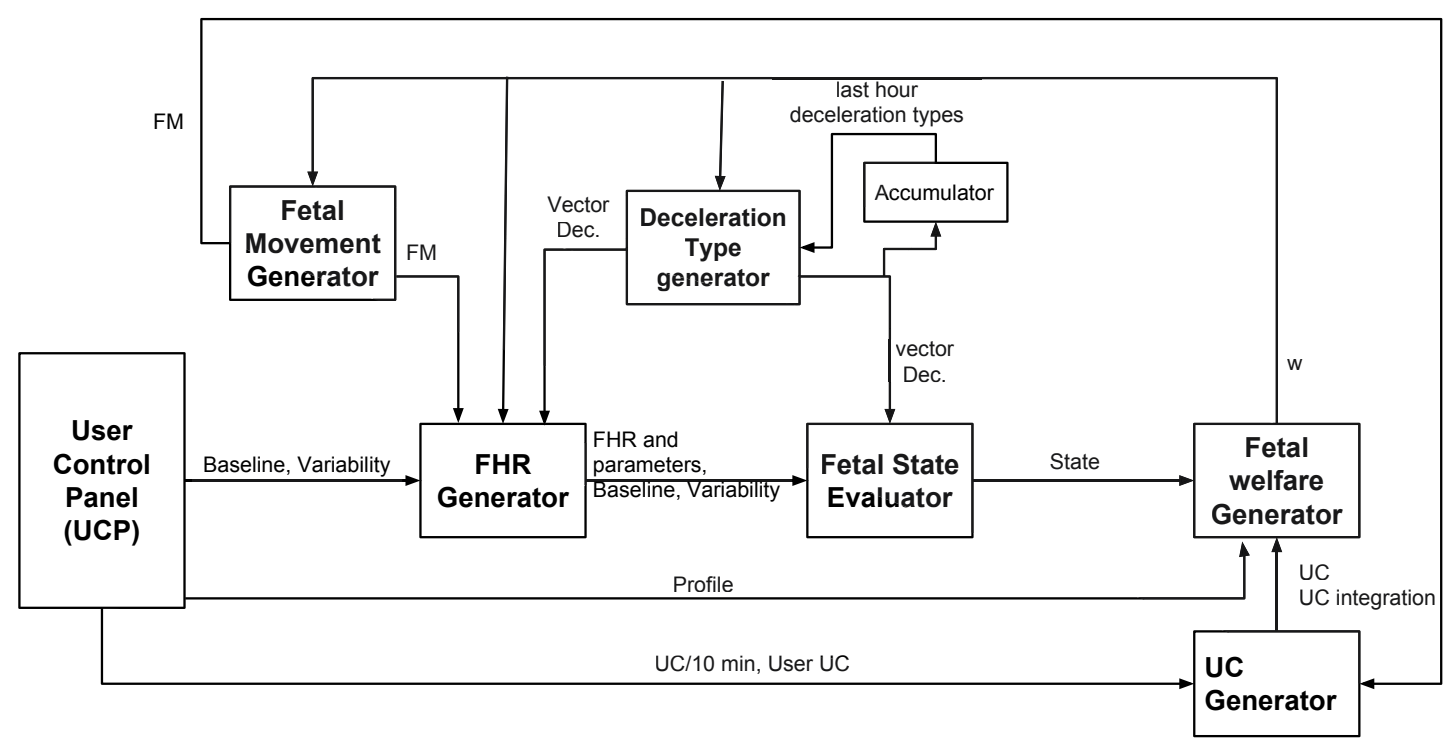

Figure 1. Block diagram showing the different subsystems of the CTG model simulator

$w$ is the welfare function which high values correspond to a bad fetal condition while low welfare function's values indicate a good fetal condition. The time constant $\tau$ takes values $\tau_{\text {charge }}$ and $\tau_{\text {discharge }}$ during or in absence of contractions, respectively. $w_{\infty}$ is the asymptotic value of $w$ and takes the value $w_{+\infty}$ during a contraction and the value $w_{-\infty}$ when no contraction is present.

The four parameters of equation (1) are dependent on the state evaluated in the fetal state evaluator block, the presence and accumulated intensity of a deceleration (through an integration operation in a sliding window of 1 hour length using a forgetting factor) and also the profile set by the user. The charge and discharge of the $w$ function are directly related to the condition of the fetus to adapt when a new CU arrives. If $\tau_{\text {charge }}$ is high and $w_{+\infty}$ is low, then when a new CU arrives the condition of the fetus is getting worst very fast. On the contrary if $\tau_{\text {charge }}$ is low and $w_{+\infty}$ is high, then when a new CU arrives the fetus is prepared for its arrival since his condition takes long time to become alarming. During the absence of $\mathrm{CU}$ something similar occurs allowing the fetus to recover faster or slower depending on the parameters' values.

\section{Results}

Figure 2 has as main objective to show, in an illustrative way, how the charging/discharging dynamics and $w_{\infty}$ values of the function $w$ acts on the dynamics changes of the FHR signal caused by two episodes of a train of consecutive UC's. The colors on the fetal welfare function $w$ show the discretized estimated states made by the state evaluator block, using the guideline of [12], and how this state
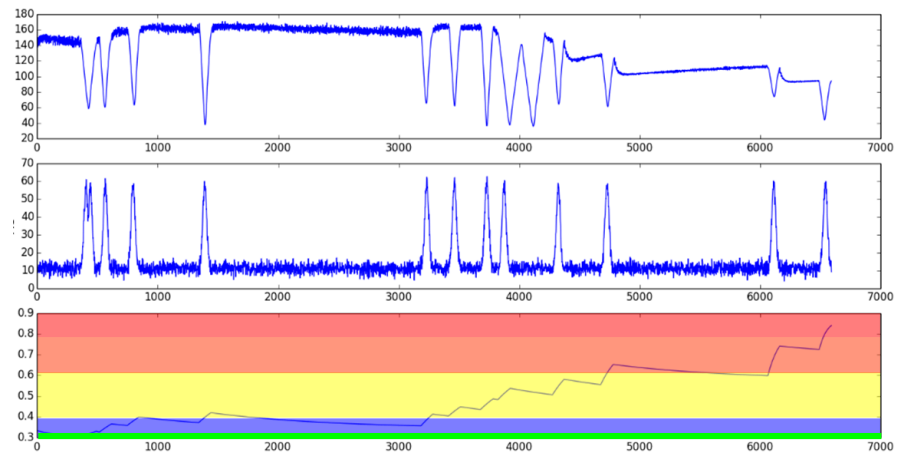

Figure 2. FHR signal, UC signal and the welfare function $w$ during a simulation under two consecutive UC's trains.

affects the parameters of the function $w$. The colors are the same used in [12] and go from green, i.e. no acidemia, to red i.e., evidence of damaging fetal asphyxia. The simulation shows that the condition of the fetus is optimal at the beginning (state in green and low $w_{-\infty}$ ). After the first train of UC, $w_{+\infty}$ and $w_{-\infty}$ change causing a progressive increase of $w . \tau_{\text {charge }}$ increases and $\tau_{\text {discharge }}$ decreases, since the estimated state of the fetus change. This results in a slower recuperation and in a worth final state before the second train of CUs arrives causing a progressive deterioration of the fetal state.

Figures 3 and 4, show two simulations obtained with the proposed model, one of 20 minutes duration and the other of 34 minutes duration. In the first simulation (Figure 3 ) the initial conditions of the fetus were optimal (120 bpm baseline, moderated variability and fetus without as- 


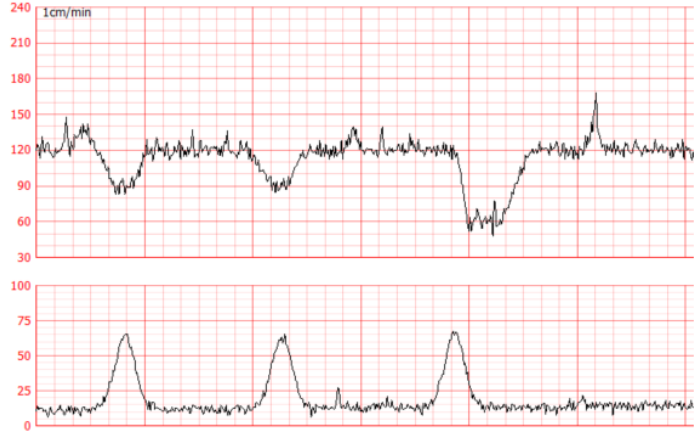

Figure 3. Simulation of a 20 minute recording of a normal CTG involving different two early and one late decelerations.

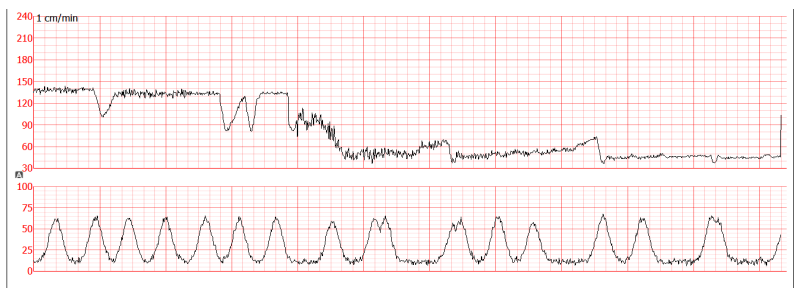

Figure 4. Simulation of a 34 minutes recording with a deterioration on fetal state due to a train of CUs.

phyxia) and no action has been performed online. The simulation results on three CUs producing two early decelerations and one late deceleration.

In the second simulation the initial conditions of the fetus concerning baseline and variability were set also optimally (140 bpm baseline and moderated variability), but the profile was set in moderated asphyxia. After a train of UCs (set online by the user) resulting in no deceleration or early deceleration, the state of the fetus starts to deteriorate, first by two late decelerations and then by a decreasing variability. This results in a prolonged deceleration, which triggers a severe decrease of the baseline.

\section{Conclusions}

The model provides the main dynamics of a CTG including baseline of the FHR, variability, accelerations, decelerations (early, late and variables). The UCP allows the user to set different simulation parameters including the possibility to generate UC or FM on-line during the simulation. This online interaction between user and model allows to change the dynamics of the fetal heart rate response starting from a chosen scenario (parameters and profile) and experimenting online how patterns evolve progressively according to the online changes that user performs.

Simulated tracings were analyzed by two experts in fetal physiology and evaluated in terms of signal realism and dynamics of the normal changes, periodic and episodic changes of the FHR with no significant differences between real and computer-generated CTG tracings.

\section{References}

[1] Nageotte MP. Fetal heart rate monitoring. Seminars in Fetal and Neonatal Medicine 2015;20(3):144-148.

[2] Ayres-de Campos D, Bernardes J. Twenty-five years after the FIGO guidelines for the use of fetal monitoring: Time for a simplified approach? International Journal of Gynecology Obstetrics 2010;110(1):1-6.

[3] Santo S, Ayres-de Campos D. Human factors affecting the interpretation of fetal heart rate tracings: an update. Current Opinion in Obstetrics and Gynecology 2012;24(2):84-88.

[4] Bastos LF, Lobo MF, van Meurs WL, Ayres-de Campos D. An intrauterine pressure generator for educational simulation of labour and delivery. Medical engineering physics 2010;32(7):740-745.

[5] McSharry PE, Clifford GD, Tarassenko L, Smith LA. A dynamical model for generating synthetic electrocardiogram signals. Biomedical Engineering IEEE Transactions on 2003;50(3):289-294.

[6] Bastos LF, Van Meurs W, Ayres-de Campos D. A model for educational simulation of the evolution of uterine contractions during labor. Computer methods and programs in biomedicine 2012;107(2):242-247.

[7] Warrick PA, Hamilton EF, Precup D, Kearney RE. Identification of the dynamic relationship between intrapartum uterine pressure and fetal heart rate for normal and hypoxic fetuses. Biomedical Engineering IEEE Transactions on 2009;56(6): 1587-1597.

[8] Warrick PA, Hamilton EF, Precup D, Kearney RE. Classification of normal and hypoxic fetuses from systems modeling of intrapartum cardiotocography. Biomedical Engineering IEEE Transactions on 2010;57(4):771-779.

[9] van der Hout-van der Jagt MB, Oei S, Bovendeerd PH. A mathematical model for simulation of early decelerations in the cardiotocogram during labor. Medical Engineering Physics 2012;34(5):579-589.

[10] van der Hout-van MB, Oei SG, Bovendeerd PH, et al. Simulation of reflex late decelerations in labor with a mathematical model. Early human development 2013;89(1):7-19.

[11] van der Hout-van MB, Jongen GJ, Bovendeerd PH, Oei SG, et al. Insight into variable fetal heart rate decelerations from a mathematical model. Early Human Development 2013; 89(6):361-369.

[12] Parer JT, Ikeda T. A framework for standardized management of intrapartum fetal heart rate patterns. American journal of obstetrics and gynecology 2007;197(1):26-e1.

Address for correspondence:

Alfredo Illanes

Facultad de Ciencias de la Ingeniería

General Lagos 2086, Campus Miraflores, Valdivia, Chile alfredoillanes@uach.cl 